\title{
RANDOM POLYNOMIALS, RANDOM MATRICES AND L-FUNCTIONS
}

\author{
DAVID W FARMER, FRANCESCO MEZZADRI, AND NINA C SNAITH
}

\begin{abstract}
We show that the Circular Orthogonal Ensemble of random matrices arises naturally from a family of random polynomials. This sheds light on the appearance of random matrix statistics in the zeros of the Riemann zeta-function.
\end{abstract}

\section{INTRODUCTION}

The statistics of eigenvalues of unitary matrices, chosen uniformly with respect to Haar measure on $U(N)$, are observed to closely match the statistics of zeros of the Riemann zetafunction and other $L$-functions [21, 22, 11, 25. In addition, eigenvalues of other compact classical matrix groups give a good model of the zeros of various families of $L$-functions [16 15, 24, 14, 8. Furthermore, the characteristic polynomials of the matrices provide a good model of the $L$-functions themselves [19, 3, 18, 4, 13.

The $L$-functions studied in number theory are Dirichlet series having a functional equation and an Euler product. In this paper we are concerned with a wider class of Dirichlet series which have a functional equation but do not have an Euler product (see, for example, 9, 10]). It has been suggested that such functions can be modelled by random self-reciprocal polynomials [9. In Appendix A we discuss the example of Epstein zeta functions. It is also possible to create Dirichlet series with a functional equation but no Euler product from Maass forms on a nonarithmetic group [7]. Since these functions do not have an Euler product, they are not expected to satisfy the Riemann hypothesis. However, it is possible that occasionally such functions will satisfy the Riemann hypotheses or, as is more likely, will have a large number of consecutive zeros, say the first 100 trillion of them, on the critical line.

This paper is motivated by the following questions: do the Riemann zeta-function and the other $L$-functions of number theory behave differently than random Dirichlet series with functional equation which just happen to satisfy the Riemann hypothesis? That is, does the Euler product have any effect on the zeros beyond forcing them to be on the critical line? Our results suggest that the answer is 'yes', and the Euler product also has an effect on the local statistics of the zeros.

1.1. Random polynomials. In random matrix theory, the characteristic polynomial of a random matrix can be viewed as a random polynomial where the randomness is explicitly encoded in the zeros. For example, consider the Weyl integration formula for the classical compact groups 28, 15] or the $\beta=1,2,4$ ensembles of random matrix theory [20]. On the

Date: September 2, 2018.

Research supported by the American Institute of Mathematics and the Focused Research Group grant (0244660) from the NSF. This work was started during the program on Random Matrix Applications in Number Theory at the Isaac Newton Institute for Mathematical Sciences. The second and third authors were also supported by a Royal Society Dorothy Hodgkin Fellowship, and the third author was partially supported by EPSRC. 
other hand, usually in the study of random polynomials (for a review of the subject see, e.g., Farahmand [6]) the randomness is explicitly encoded in the coefficients of the polynomials. We will show that there is a simple but surprising connection between these two perspectives, and we believe this connection is relevant to the appearance of random matrix statistics in the zeros of the Riemann zeta-function and other $L$-functions.

The polynomials we consider are of the form

$$
f(z)=z^{N}+a_{1} z^{N-1}+\cdots+a_{N}
$$

with $\left|a_{N}\right|=1$ which have the symmetry

$$
f(z)=a_{N} z^{N} \bar{f}\left(\frac{1}{z}\right),
$$

where $\bar{f}(z):=\overline{f(\bar{z})}$. Such polynomials are called self-reciprocal. Equation (1.2) ensures that the zeros of $f$ occur either on the unit circle or in pairs located symmetrically with respect to the unit circle. The symmetry (1.2) is analogous to the functional equation of $L$-functions which arise in number theory, see (1.12) below. In terms of the coefficients $a_{j}$ the functional equation (1.2) translates into the relation

$$
a_{N-j}=a_{N} \bar{a}_{j} .
$$

The change of variables $z=e^{i x}$ in the function $a_{N}^{-\frac{1}{2}} z^{-N / 2} f(z)$ converts a self-reciprocal algebraic polynomial into a real trigonometric polynomial

$$
\sum_{0 \leq n \leq N / 2} c_{n} \cos ((N / 2-n) x)+d_{n} \sin ((N / 2-n) x)
$$

where $c_{n}$ and $d_{n}$ are real. And if $f$ has real coefficients then the associated trigonometric polynomial is an even function of $x$, having only cosine terms in its expansion. The roots of these trigonometric polynomials are either on the real line or in complex conjugate pairs. This is somewhat closer to the symmetry of an $L$-function, but in this paper we phrase everything in terms of algebraic polynomials (1.1) to emphasize the comparison with characteristic polynomials of random matrices.

For even trigonometric polynomials, whose coefficients are independent standard normal random variables, Dunnage [5] discovered that the expected number of real zeros is given by

$$
\frac{2 N}{\sqrt{3}}+O\left(N^{11 / 13}(\log N)^{3 / 13}\right)
$$

Bogomolny et al. 2 extended Dunnage's result and studied the average fraction of roots of the polynomial (1.1) on the unit circle when $a_{N}=1$ and the coefficients $a_{j}$ (for $1 \leq j<N / 2$ ) are complex normal random variables with mean zero and whose standard deviation $\sigma$ varies with $N$ as

$$
\sigma=\epsilon / \sqrt{N} \text {. }
$$

They discovered that in the limit $\epsilon \rightarrow 0$, i.e. when all the coefficients have a narrow distribution centered around zero, there is a small neighbourhood of $\epsilon$ where the average fraction of roots lying on the unit circle is one. Instead, when the coefficients have a broad distribution, i.e. in the limit $\epsilon \rightarrow \infty$, the average fraction of zeros on the unit circle tends to $1 / \sqrt{3}$. They also computed the two-point correlation function of the zeros on the unit circle, which for short distances grows linearly. 
1.2. $L$-functions. An $L$-function is a Dirichlet series,

$$
L(s)=\sum_{n=1}^{\infty} \frac{a_{n}}{n^{s}},
$$

with $a_{n}=O_{\epsilon}\left(n^{\epsilon}\right)$ for every $\epsilon>0$, which has an analytic continuation to the complex plane (except for possible poles on the line Res $=1$ ) along with two additional properties. First, it has a functional equation

$$
\xi_{L}(s):=\gamma_{L}(s) L(s)=\varepsilon \overline{\xi_{L}}(1-s),
$$

with $|\varepsilon|=1$ and $\gamma_{L}$ of the form

$$
\gamma_{L}(s)=P(s) Q^{s} \prod_{j=1}^{w} \Gamma\left(w_{j} s+\mu_{j}\right),
$$

where $Q>0, w_{j}>0, \operatorname{Re} \mu_{j} \geq 0$, and $P$ is a polynomial whose only zeros in $\sigma>0$ are at the poles of $L(s)$. Second, it has an Euler product representation of the form

$$
L(s)=\prod_{p} L_{p}\left(1 / p^{s}\right),
$$

where the product is over the primes $p$, and

$$
L_{p}\left(1 / p^{s}\right)=\sum_{k=0}^{\infty} \frac{a_{p^{k}}}{p^{k s}}=\exp \left(\sum_{k=1}^{\infty} \frac{b_{p^{k}}}{p^{k s}}\right),
$$

where $b_{n}=O\left(n^{\theta}\right)$ with $\theta<\frac{1}{2}$. It is conjectured that such $L$-functions satisfy the Riemann hypothesis, which is the assertion that the nontrivial zeros lie on the critical line $\operatorname{Re}(s)=\frac{1}{2}$.

The functional equation is a symmetry with respect to the line $\operatorname{Re}(s)=\frac{1}{2}$, so the nontrivial zeros of $L$ are on the line $\operatorname{Re}(s)=\frac{1}{2}$, or they are located symmetrically on either side of it. To show the analogy with self-reciprocal polynomials (1.2) it is more convenient to write the functional equation in asymmetric form:

$$
L(s)=\varepsilon X_{L}(s) \bar{L}(1-s),
$$

where $X_{L}(s)=\overline{\gamma_{L}}(1-s) / \gamma_{L}(s)$. Note that $\left|X_{L}\right|=1$ on the $\frac{1}{2}$-line, that is, on the line of symmetry of the $L$-function. Thus, there is a perfect analogy between the self-reciprocal property of $f$ and the functional equation of $L$.

1.3. Results and discussion. For Dirichlet series with a functional equation but no Euler product, it is not expected that all zeros lie on the critical line. However, it is possible that even if such a Dirichlet series does not satisfy the Riemann hypothesis, it could have, say, its first 100 trillion zeros on the critical line. Our motivating question is this: is the Riemann zeta-function, with its Euler product, distinguishable from a random Dirichlet series with functional equation that just happens to have its first 100 trillion zeros on the critical line? By considering the analogous case of random self-reciprocal polynomials, we suggest that the answer is 'yes'.

Let us consider the space $\mathcal{R}_{N}$ of all the self-reciprocal polynomials of degree $N$. Given a nonvanishing probability distribution on $\mathcal{R}_{N}$ (meaning that nonempty open sets have positive measure) the subset $\mathcal{C}_{N}$ of those polynomials which happen to have all of their zeros on the unit circle has positive measure. The proof is quite simple. The zeros of a self-reciprocal polynomial lie either on the unit circle or in pairs symmetric with respect to the unit circle, 
and they are continuous functions of the coefficients. Two zeros on the circle must "collide" in order to move off the circle, so there is a small open neighborhood of coefficients in which the zeros remain on the circle. This neighbourhood has positive measure. Therefore, the restriction to $\mathcal{C}_{N}$ of the measure on $\mathcal{R}_{N}$ is unique and well defined. Such a restriction is then made into a probability measure by renormalizing the volume of $\mathcal{C}_{N}$ to one.

Our interest is mainly concentrated on the distribution of the roots of those polynomials whose zeros lie all on the unit circle. Therefore, our approach will be to define a joint probability density function on the coefficients of the polynomials in $\mathcal{R}_{N}$ and to study its restriction to $\mathcal{C}_{N}$. The coefficients $a_{n}$ of polynomials with all their zeros on the circle are bounded in magnitude by $\left(\begin{array}{l}N \\ n\end{array}\right)$, therefore not only has $\mathcal{C}_{N}$ positive measure, but it is also compact. Thus, the most natural choice is to put a distribution on the coefficients $a_{n}$ which is uniform on a bounded disk containing $\mathcal{C}_{N}$ and zero outside. The following theorem gives the joint probability density function for the roots of such polynomials. Petersen and Sinclair [23] have found some interesting geometric properties of the coefficients of these polynomials. They have also proved independently a result very similar to Theorem 1.1. In their work (Lemma 4.1 of [23]) they fix the coefficient $a_{N}$, rather than allowing it to vary, but they arrive at the same Vandermonde form for the Jacobian.

In what follows we denote by $\Delta\left(x_{1}, \ldots, x_{N}\right)$ the Vandermonde determinant, i.e.

$$
\Delta\left(x_{1}, \ldots, x_{N}\right)=\prod_{j<k}\left(x_{k}-x_{j}\right)
$$

and we denote by $e_{n}$ the $n$th elementary symmetric function

$$
e_{n}\left(x_{1}, \ldots, x_{N}\right)=\sum_{1 \leq i_{1}<\cdots<i_{n} \leq N} x_{i_{1}} \cdots x_{i_{n}} .
$$

Theorem 1.1. Suppose $N$ is odd. Consider random monic polynomials $z^{N}+\sum_{n=1}^{N} a_{n} z^{N-n}$ satisfying the self-reciprocal property $a_{N-n}=a_{N} \bar{a}_{n}$, with $a_{1}, \ldots, a_{(N-1) / 2}$ chosen independently and uniformly in $\left|a_{n}\right| \leq\left(\begin{array}{c}N \\ n\end{array}\right)$, and with $\phi$ chosen uniformly in $[0,2 \pi)$, where $a_{N}=e^{i \phi}$, and restrict to those polynomials having all zeros on the unit circle. The joint probability density function of the set of zeros $e^{i \delta_{1}}, \ldots, e^{i \delta_{N}}$ is given, up to a normalization constant, by

$$
\left|\Delta\left(e^{i \delta_{1}}, \ldots, e^{i \delta_{N}}\right)\right|=\prod_{j<k}\left|e^{i \delta_{k}}-e^{i \delta_{j}}\right| .
$$

For $N$ even, consider self-reciprocal random monic polynomials with $a_{1}, \ldots, a_{N / 2}$ chosen independently and uniformly in $\left|a_{n}\right| \leq\left(\begin{array}{l}N \\ n\end{array}\right)$, and restrict to those polynomials having all zeros on the unit circle. The joint probability density function for the set of zeros $e^{i \delta_{1}}, \ldots, e^{i \delta_{N}} i s$ given, up to a normalization constant, by

$$
\left|e_{N / 2}\left(e^{i \delta_{1}}, \ldots, e^{i \delta_{N}}\right) \Delta\left(e^{i \delta_{1}}, \ldots, e^{i \delta_{N}}\right)\right|=\left|e_{N / 2}\left(e^{i \delta_{1}}, \ldots, e^{i \delta_{N}}\right)\right| \prod_{j<k}\left|e^{i \delta_{k}}-e^{i \delta_{j}}\right|,
$$

where $e_{N / 2}\left(e^{i \delta_{1}}, \ldots, e^{i \delta_{N}}\right)$ is the $N / 2$ elementary symmetric function in the variables $e^{i \delta_{1}}, \ldots, e^{i \delta_{N}}$ and is equal to $(-1)^{N / 2}$ times the coefficient $a_{N / 2}$.

In particular, the joint probability density function for odd $N$ is the same as that for eigenvalues of a randomly chosen matrix in the Circular Orthogonal Ensemble COE $(N)$.

Some aspects of this result can be derived from [27] but they approach the subject from a different perspective. 
The theorem suggests that if a Dirichlet series with functional equation is chosen at random, and all of the zeros in a particular interval of the critical strip happen to lie on the critical line, then those zeros should have similar statistics to those of eigenvalues of matrices from the COE. The COE is the symmetric space $U(N) / O(N)$ with the measure induced from Haar measure on $U(N)$. Thus the joint probability density function for the eigenvalues is

$$
\frac{1}{(4 \sqrt{\pi})^{N} \Gamma(1+N / 2)}\left|\Delta\left(e^{i \delta_{1}}, \ldots, e^{i \delta_{N}}\right)\right|=\frac{1}{(4 \sqrt{\pi})^{N} \Gamma(1+N / 2)} \prod_{j<k}\left|e^{i \delta_{k}}-e^{i \delta_{j}}\right| .
$$

All numerical calculations of zeros of $L$-functions having an Euler product show the statistics of the CUE (the group $U(N)$ with Haar measure), so this suggests that the $L$-functions from number theory are not typical Dirichlet series with a functional equation.

Thus, the Euler product which is considered a necessary condition for a Dirichlet series with functional equation to satisfy the Riemann hypothesis does more then just force the zeros onto the critical line. The Euler product fundamentally changes the nature of the spacing of the zeros, in particular changing the linear repulsion of zeros of random polynomials and the COE into the quadratic repulsion of the CUE, whose joint probability density function is given by

$$
\frac{1}{(2 \pi)^{N} N !}\left|\Delta\left(e^{i \delta_{1}}, \ldots, e^{i \delta_{N}}\right)\right|^{2}=\frac{1}{(2 \pi)^{N} N !} \prod_{j<k}\left|e^{i \delta_{k}}-e^{i \delta_{j}}\right|^{2} .
$$

In random matrix theory it has long been conjectured that in the limit $N \rightarrow \infty$ the local correlations of the eigenvalues of random matrices depend exclusively on the invariance properties of the probability distribution that defines the ensemble and not on the explicit form of the measure itself. This random matrix hypothesis is one of the most important features of the subject. Mathematically it translates into the statement that, provided that the local eigenvalue density has the same asymptotic behaviour, the local correlations are mainly determined by the absolute value of (powers) of the Vandermonde, whose origin is essentially geometrical. Therefore, we conjecture that in the limit $N \rightarrow \infty$ the local statistics of the roots of the polynomials in $\mathcal{C}_{N}$ will be independent of our choice of the joint probability density function for the coefficients of the polynomials in $\mathcal{R}_{N}$. Indeed, because of this reason the extra factor $\left|e_{N / 2}\right|$ appearing in equation (1.16) of theorem 1.1] when $N$ is even should not affect the local correlations of the roots in the limit $N \rightarrow \infty$.

Bogomolny et al. 2] studied self-reciprocal polynomials whose coefficients are independent complex normal random variables and computed the two-point correlation function $R_{2}(\delta)$ of the subset of zeros that lie on the unit circle. As $\delta \rightarrow 0$ they observed linear repulsion between the arguments of the zeros. Such level repulsion is a direct consequence of the Vandermonde that appears in equations (1.15) and (1.16) of theorem 1.1 (or more appropriately for their case in equations (3.1) and (3.2) of lemma 3.1) and supports our conjecture.

The appearance of CUE statistics for arithmetic $L$-functions has been compared to the appearance of CUE statistics in a chaotic system without time-reversal symmetry [17, 1]. Indeed, the appearance of the CUE statistics for zeros of $L$-functions has been heuristically explained by the analogy between the periodic orbit sum for the density of states of a classically chaotic system with no time-reversal symmetry and the density of zeros of the Riemann zeta function expressed as a sum over primes. Our observation on the effect of the Euler product can be viewed as further evidence for that point of view. 
In their article Bogomolny et al. [2] commented that the linear behaviour of $R_{2}(\delta)$ in the limit $\delta \rightarrow 0$ was surprising, because it is typical of quantum mechanical systems whose dynamics is invariant under time reversal. Instead, they would have expected quadratic repulsion between the roots lying on the circle, which is characterizes chaotic systems whose dynamics is not time reversal invariant and that self-reciprocal polynomials with arbitrary complex coefficients were expected to model. In the case that we study we are facing the same paradox. We are modelling $L$-functions having a functional equation but no Euler product and observe that the zeros lying on the critical line should have the same local correlations as the eigenvalues of matrices in the COE ensemble. Instead, the zeros on the critical line of $L$-functions that have the further constraint of being expressed in terms of an Euler product are correlated like the eigenvalues of matrices in the CUE ensemble. The natural expectation would be the opposite, since matrices in the CUE ensemble are not in any way restricted, except that the measure on their set should be invariant under group multiplication. The COE ensemble is obtained by imposing extra symmetry constraints on the matrices in the CUE, whose set, therefore, includes the set of matrices forming the COE.

We also consider the case of real self-reciprocal polynomials. That is, polynomials of the form (1.1) satisfying (1.2), where the $a_{n}$ are real. These polynomials have their zeros in complex conjugate pairs, and in particular the zeros near $z=1$ would be expected to have somewhat different behavior than the bulk of the zeros. Anomalous behavior of the low-lying zeros occurs for families of arithmetic $L$-functions, and this behavior has been modelled by the low-lying eigenvalues of matrices from the classical Symplectic and Orthogonal groups.

Theorem 1.2. Let $N=2 M$. Consider random real monic polynomials $z^{N}+\sum_{n=1}^{N} a_{n} z^{N-n}$ satisfying the self-reciprocal property $a_{n}=a_{N-n}$, with $a_{n}$ chosen independently and uniformly in the interval $\left|a_{n}\right| \leq\left(\begin{array}{l}N \\ n\end{array}\right)$, and restrict to those polynomials having all zeros on the unit circle. The joint probability density function of the set of zeros $e^{i t_{1}}, e^{-i t_{1}}, \ldots, e^{i t_{M}}, e^{-i t_{M}}$ is given, up to a normalization constant, by

$$
\prod_{m}\left|e^{i t_{m}}-e^{-i t_{m}}\right| \prod_{j<k}\left|e^{i t_{k}}-e^{i t_{j}}\right|\left|e^{i t_{k}}-e^{-i t_{j}}\right| .
$$

In the case where the polynomial has odd degree $N=2 M+1$ with roots $-1, e^{i t_{1}}, e^{-i t_{1}}, \ldots$, $e^{i t_{M}}, e^{-i t_{M}}$ then the joint probability density function of $e^{i t_{1}}, e^{-i t_{1}}, \ldots, e^{i t_{M}}, e^{-i t_{M}}$ is the same as given above.

The above measure can be written as, up to a normalization constant,

$$
\left|\prod_{m} \sin \left(t_{m}\right) \prod_{j<k} \sin \left(\frac{t_{k}-t_{j}}{2}\right) \sin \left(\frac{t_{k}+t_{j}}{2}\right)\right| .
$$

We note that this is the square root of Haar measure of $U S p(N)$. Thus, random real selfreciprocal polynomials, restricted to have all their zeros on the unit circle, do show anomalous spacings in their low lying zeros. But it is not the same anomalous spacing that has previously been found in families of arithmetic $L$-functions. This suggests that for the low-lying zeros of a family of $L$-functions with real coefficients, the Euler product has an effect on the vertical spacing of the zeros, and those $L$-functions behave differently than random real Dirichlet series with functional equation which just happen to have their first few zeros on the critical line. 
In the next section we prove the Theorems and in the following sections we give the Jacobian calculations required in the proofs. We thank Christopher Sinclair for helpful information.

\section{Proofs of THE THEOREMS}

We are given a measure on a set of polynomials described in terms of the coefficients of the polynomial, and we wish to describe the measure in terms of the roots of the polynomial. Therefore we must compute the Jacobian of the change of variables from the coefficients to the roots. It is well known that the Jacobian is the Vandermonde in the roots in the case of polynomials with real coefficients, and the Vandermonde squared in the case of complex coefficients. Thus we expect the Jacobian to be close to a Vandermonde in the case of selfreciprocal polynomials, but we were unable to find all the results we needed in the literature, so we give a complete proof below.

If $X$ is a random variable then we let $\langle X\rangle$ denote the expected value of $X$. In our case $f$ will be a random polynomial and $X=M[f]$ is some function of $f$, and we will need to compute $\langle M[f]\rangle$.

In Theorem 1.1. consider $N$ even so $a_{1}, \ldots, a_{N / 2}$ determine $f$. If $\rho_{\text {coeffs }}\left(a_{1}, \ldots, a_{N / 2}\right)$ is a probability measure on the coefficients of $f$, which is supported on the set $S$, then

$$
\langle M[f]\rangle=\int_{S} M[f] \rho_{\mathrm{coeffs}}\left(a_{1}, \ldots, a_{N / 2}\right) d a_{1} \cdots d a_{N / 2},
$$

and by definition $\int_{S} \rho_{\text {coeffs }}\left(a_{1}, \ldots, a_{N / 2}\right) d a_{1} \cdots d a_{N / 2}=1$.

We can express $\langle M[f]\rangle$ in terms of the zeros $z_{1}, \ldots, z_{N}$ of $f$. Set

$$
\rho_{\text {zeros }}\left(z_{1}, \ldots, z_{N}\right)=\rho_{\text {coeffs }}\left(a_{1}\left(z_{1}, \ldots, z_{N}\right), \ldots, a_{N}\left(z_{1}, \ldots, z_{N}\right)\right)
$$

and let $J_{\mathbb{C}}\left(z_{1}, \ldots, z_{N}\right)$ be the Jacobian of the transformation from the coefficients to the zeros. Then

$$
\langle M[f]\rangle=\int_{S^{\prime}} M[f] \rho_{\text {zeros }}\left(z_{1}, \ldots, z_{N}\right)\left|J_{\mathbb{C}}\left(z_{1}, \ldots, z_{N}\right)\right| d z_{1} \cdots d z_{N},
$$

where $S^{\prime}$ is the image of $S$ under the coordinate change. To prove the Theorems we merely specialize this discussion to our particular cases.

Proof of Theorem 1.1. We have that $\rho_{\text {coeffs }}$ is constant and $S$ is the set of coefficients of selfreciprocal polynomials having all their zeros on the unit circle. So $\rho_{\text {zeros }}$ is also constant and $S^{\prime}=S^{1} \times \cdots \times S^{1}$ where $S^{1}$ is the unit circle. Thus, we only require the Jacobian of the transformation, which is given in the following Lemma.

Lemma 2.1. If the self-reciprocal polynomial $f(z)=z^{N}+\sum_{n=1}^{N} a_{n} z^{N-n}$ has all its zeros on the unit circle, $e^{i \delta_{1}}, \ldots, e^{i \delta_{N}}$, then when $N$ is odd, the absolute value of the Jacobian of the transformation from coefficient variables $\operatorname{Re} a_{1}, \operatorname{Im} a_{1}, \ldots, \operatorname{Re} a_{(N-1) / 2}, \operatorname{Im} a_{(N-1) / 2}, \phi$ (where $\left.a_{N}=e^{i \phi}\right)$ to the zero variables $\delta_{1}, \ldots, \delta_{N}$ is given by

$$
\left|J_{\mathbb{C}}\left(e^{i \delta_{1}}, \ldots, e^{i \delta_{N}}\right)\right|=2^{-(N-1) / 2}\left|\Delta\left(e^{i \delta_{1}}, \ldots, e^{i \delta_{N}}\right)\right| .
$$


When $N$ is even, the absolute value of the Jacobian of the transformation from coefficients $\operatorname{Re} a_{1}, \operatorname{Im} a_{1}, \ldots, \operatorname{Re} a_{N / 2}, \operatorname{Im} a_{N / 2}$ to zeros $\delta_{1}, \ldots, \delta_{N}$ is given by

$$
\left|J_{\mathbb{C}}\left(e^{i \delta_{1}}, \ldots, e^{i \delta_{N}}\right)\right|=2^{-\frac{N}{2}}\left|e_{N / 2}\left(e^{i \delta_{1}}, \ldots, e^{i \delta_{N}}\right) \Delta\left(e^{i \delta_{1}}, \ldots, e^{i \delta_{N}}\right)\right|,
$$

where $e_{N / 2}\left(e^{i \delta_{1}}, \ldots, e^{i \delta_{N}}\right)$ is the $N / 2$ elementary symmetric function.

A proof of the Lemma can be found in Section 3.

Assembling the pieces we have, for example for odd $N$,

$$
\langle M[f]\rangle=\frac{1}{(4 \sqrt{\pi})^{N} \Gamma(1+N / 2)} \int_{0}^{2 \pi} \cdots \int_{0}^{2 \pi} M[f]\left|\Delta\left(e^{i \delta_{1}}, \ldots, e^{i \delta_{N}}\right)\right| d \delta_{1} \cdots d \delta_{N},
$$

which is equivalent to Theorem 1.1 .

The proof of Theorem 1.2 is identical except that we require the following lemma, which is proven in Section 4.

Lemma 2.2. If the real self-reciprocal polynomial $f$ has even degree $N=2 M$ and has all its zeros on the unit circle, then the absolute value of the Jacobian of the transformation from coefficients $a_{1}, \ldots, a_{M}$ to zeros $e^{i t_{1}}, e^{-i t_{1}}, \ldots, e^{i t_{M}}, e^{-i t_{M}}$ is given by

$$
\left|J_{\mathbb{R}}\left(e^{i t_{1}}, e^{-i t_{1}}, \ldots, e^{i t_{M}}, e^{-i t_{M}}\right)\right|=\left|\prod_{m}\left(e^{i t_{m}}-e^{-i t_{m}}\right) \prod_{j<k}\left(e^{i t_{k}}-e^{i t_{j}}\right)\left(e^{i t_{k}}-e^{-i t_{j}}\right)\right| .
$$

In the case the degree $N=2 M+1$ is odd, and $f$ has zeros at $-1, e^{i t_{1}}, e^{-i t_{1}}, \ldots, e^{i t_{M}}, e^{-i t_{M}}$, the Jacobian is again given by the above formula.

\section{Calculation of the Jacobian: complex Case}

We prove the following generalization of Lemma 2.1

Lemma 3.1. Let the roots of a self-reciprocal polynomial $f$ be $\alpha_{1}=e^{i \delta_{1}}, \ldots, \alpha_{L}=e^{i \delta_{L}}$, for those roots on the unit circle, and $\beta_{1}=\rho_{1} e^{i \theta_{1}}, \frac{1}{\bar{\beta}_{1}}=\frac{e^{i \theta_{1}}}{\rho_{1}}, \ldots, \beta_{M}=\rho_{M} e^{i \theta_{M}}, \frac{1}{\bar{\beta}_{M}}=\frac{e^{i \theta_{M}}}{\rho_{M}}$ for the roots occurring in pairs off the unit circle. When $N=L+2 M$ is odd, the absolute value of the Jacobian of the transformation from coefficients $\operatorname{Re} a_{1}, \operatorname{Im} a_{1}, \ldots, \operatorname{Re} a_{(N-1) / 2}, \operatorname{Im} a_{(N-1) / 2}, \phi$ (where $a_{N}=e^{i \phi}$ ) to zeros $\rho_{1}, \theta_{1}, \rho_{2}, \theta_{2}, \ldots, \rho_{M}, \theta_{M}, \delta_{1}, \ldots, \delta_{L}$ is given by

$$
\begin{aligned}
\left|J_{\mathbb{C}}\left(\rho_{1}, \theta_{1}, \rho_{2}, \theta_{2}, \ldots, \rho_{M}, \theta_{M}, \delta_{1}, \ldots, \delta_{L}\right)\right| \\
=2^{M-(N-1) / 2}\left|\left(\prod_{m=1}^{M} \frac{1}{\rho_{m}}\right) \Delta\left(\beta_{1}, \frac{1}{\bar{\beta}_{1}}, \beta_{2}, \frac{1}{\bar{\beta}_{2}}, \ldots, \beta_{m}, \frac{1}{\bar{\beta}_{m}}, \alpha_{1}, \ldots, \alpha_{L}\right)\right| .
\end{aligned}
$$

When $N=L+2 M$ is even, the absolute value of the Jacobian of the transformation from coefficients $\operatorname{Re} a_{1}, \operatorname{Im} a_{1}, \ldots, \operatorname{Re} a_{N / 2}, \operatorname{Im} a_{N / 2}$ to zeros $\rho_{1}, \theta_{1}, \rho_{2}, \theta_{2}, \ldots, \rho_{M}, \theta_{M}, \delta_{1}, \ldots, \delta_{L}$ is given by

$$
\begin{aligned}
\left|J_{\mathbb{C}}\left(\rho_{1}, \theta_{1}, \rho_{2}, \theta_{2}, \ldots, \rho_{M}, \theta_{M}, \delta_{1}, \ldots, \delta_{L}\right)\right| \\
=2^{M-\frac{N}{2}} \mid\left(\prod_{m=1}^{M} \frac{1}{\rho_{m}}\right) e_{N / 2}\left(\beta_{1}, \frac{1}{\bar{\beta}_{1}}, \beta_{2}, \frac{1}{\bar{\beta}_{2}}, \ldots, \beta_{m}, \frac{1}{\bar{\beta}_{m}}, \alpha_{1}, \ldots, \alpha_{L}\right) \\
\quad \times \Delta\left(\beta_{1}, \frac{1}{\bar{\beta}_{1}}, \beta_{2}, \frac{1}{\bar{\beta}_{2}}, \ldots, \beta_{m}, \frac{1}{\bar{\beta}_{m}}, \alpha_{1}, \ldots, \alpha_{L}\right) \mid,
\end{aligned}
$$

where the $e_{N / 2}$ is the $N / 2$ elementary symmetric function. 
Proof. The polynomial $f(z)$ has order $N=L+2 M$ :

$$
\begin{aligned}
f(z) & =\left(z-\alpha_{1}\right)\left(z-\alpha_{2}\right) \cdots\left(z-\alpha_{L}\right)\left(z-\beta_{1}\right)\left(z-\frac{1}{\bar{\beta}_{1}}\right) \cdots\left(z-\beta_{M}\right)\left(z-\frac{1}{\bar{\beta}_{M}}\right) \\
& =z^{N}+a_{1} z^{N-1}+a_{2} z^{N-2}+\cdots+a_{N-2} z^{2}+a_{N-1} z+a_{N} .
\end{aligned}
$$

Note that if we define

$$
\begin{array}{rlrl}
\alpha_{j} & =e^{i \delta_{j}}, & j & =1, \ldots, L \\
\beta_{j} & =t_{j} \rho_{j}=e^{i \theta_{j}} \rho_{j}, & j & =1, \ldots, M \\
\frac{1}{\bar{\beta}_{j}} & =\frac{t_{j}}{\rho_{j}}=\frac{e^{i \theta_{j}}}{\rho_{j}}, & j & j, \ldots, M
\end{array}
$$

(with $\delta_{j}, \rho_{j}$ and $\theta_{j}$ real) then $a_{N}$ is on the unit circle and

$$
a_{N}=(-1)^{N} e^{2 i \theta_{1}} e^{2 i \theta_{2}} \cdots e^{2 i \theta_{M}} e^{i \delta_{1}} \cdots e^{i \delta_{L}}=e^{i \phi} .
$$

Also

$$
a_{j}=(-1)^{j} e_{j}\left(\alpha_{1}, \ldots, \alpha_{L}, \beta_{1}, \frac{1}{\bar{\beta}_{1}}, \ldots, \beta_{M}, \frac{1}{\bar{\beta}_{M}}\right),
$$

where $e_{j}\left(x_{1}, \ldots, x_{n}\right)$ is the $j$ th elementary symmetric function. In addition we have

$$
a_{N-j}=a_{N} \bar{a}_{j}
$$

since by construction $f$ is self-reciprocal.

For now we take $N$ odd; the slight variation when $N$ is even is described at the end of this section. We want the Jacobian of the transformation from the independent real variables $\operatorname{Re} a_{1}, \operatorname{Im} a_{1}, \ldots, \operatorname{Re} a_{\frac{N-1}{2}}, \operatorname{Im} a_{\frac{N-1}{2}}, \phi$ to the real independent variables $\rho_{1}, \theta_{1}, \rho_{2}, \theta_{2}$, $\ldots, \rho_{M}, \theta_{M}, \delta_{1}, \ldots, \delta_{L}$.

So, the Jacobian is:

$$
\begin{aligned}
& J_{\mathbb{C}}=\left|\begin{array}{cccccccc}
\frac{\partial \operatorname{Re} a_{1}}{\partial \rho_{1}} & \frac{\partial \operatorname{Re} a_{1}}{\partial \theta_{1}} & \cdots & \frac{\partial \operatorname{Re} a_{1}}{\partial \rho_{M}} & \frac{\partial \operatorname{Re} a_{1}}{\partial \theta_{M}} & \frac{\partial \operatorname{Re} a_{1}}{\partial \delta_{1}} & \cdots & \frac{\partial \operatorname{Re} a_{1}}{\partial \delta_{L}} \\
\frac{\partial \operatorname{Im} a_{1}}{\partial \rho_{1}} & \frac{\partial \operatorname{Im} a_{1}}{\partial \theta_{1}} & \cdots & \frac{\partial \operatorname{Im} a_{1}}{\partial \rho_{M}} & \frac{\partial \operatorname{Im} a_{1}}{\partial \theta_{M}} & \frac{\partial \operatorname{Im} a_{1}}{\partial \delta_{1}} & \cdots & \frac{\partial \operatorname{Im} a_{1}}{\partial \delta_{L}} \\
\vdots & \vdots & \ddots & \vdots & \vdots & \vdots & \ddots & \vdots \\
\frac{\partial \operatorname{Re} a_{\frac{N-1}{2}}}{\partial \rho_{1}} & \frac{\partial \operatorname{Re} a_{\frac{N-1}{2}}}{\partial \theta_{1}} & \cdots & \frac{\partial \operatorname{Re} a_{\frac{N-1}{2}}}{\partial \rho_{M}} & \frac{\partial \operatorname{Re} a_{\frac{N-1}{2}}}{\partial \theta_{M}} & \frac{\partial \operatorname{Re} a_{\frac{N-1}{2}}}{\partial \delta_{1}} & \cdots & \frac{\partial \operatorname{Re} a_{\frac{N-1}{2}}}{\partial \delta_{L}} \\
\frac{\partial \operatorname{Im} a_{\frac{N-1}{2}}}{\partial \rho_{1}} & \frac{\partial \operatorname{Im} a_{\frac{N-1}{2}}}{\partial \theta_{1}} & \cdots & \frac{\partial \operatorname{Im} a_{\frac{N-1}{2}}}{\partial \rho_{M}} & \frac{\partial \operatorname{Im} a_{\frac{N-1}{2}}}{\partial \theta_{M}} & \frac{\partial \operatorname{Im} a_{\frac{N-1}{2}}}{\partial \delta_{1}} & \cdots & \frac{\partial \operatorname{Im} a_{\frac{N-1}{2}}}{\partial \delta_{L}} \\
\frac{\partial \phi}{\partial \rho_{1}} & \frac{\partial \phi}{\partial \theta_{1}} & \cdots & \frac{\partial \phi}{\partial \rho_{M}} & \frac{\partial \phi}{\partial \theta_{M}} & \frac{\partial \phi}{\partial \delta_{1}} & \cdots & \frac{\partial \phi}{\partial \delta_{L}}
\end{array}\right| \\
& =\left(-\frac{1}{2}\right)^{\frac{N-1}{2}}\left|\begin{array}{cccccccc}
\frac{\partial a_{1}}{\partial \rho_{1}} & \frac{\partial a_{1}}{\partial \theta_{1}} & \cdots & \frac{\partial a_{1}}{\partial \rho_{M}} & \frac{\partial a_{1}}{\partial \theta_{M}} & \frac{\partial a_{1}}{\partial \delta_{1}} & \cdots & \frac{\partial a_{1}}{\partial \delta_{L}} \\
\frac{\partial \bar{a}_{1}}{\partial \rho_{1}} & \frac{\partial \bar{a}_{1}}{\partial \theta_{1}} & \cdots & \frac{\partial \bar{a}_{1}}{\partial \rho_{M}} & \frac{\partial \bar{a}_{1}}{\partial \theta_{M}} & \frac{\partial \bar{a}_{1}}{\partial \delta_{1}} & \cdots & \frac{\partial \bar{a}_{1}}{\partial \delta_{L}} \\
\vdots & \vdots & \ddots & \vdots & \vdots & \vdots & \ddots & \vdots \\
\frac{\partial a_{\frac{N-1}{2}}}{\partial \rho_{1}} & \frac{\partial a_{\frac{N-1}{2}}}{\partial \theta_{1}} & \cdots & \frac{\partial a_{\frac{N-1}{2}}}{\partial \rho_{M}} & \frac{\partial a_{\frac{N-1}{2}}}{\partial \theta_{M}} & \frac{\partial a_{\frac{N-1}{2}}}{\partial \delta_{1}} & \cdots & \frac{\partial a_{\frac{N-1}{2}}}{\partial \delta_{L}} \\
\frac{\partial \bar{a}_{\frac{N-1}{2}}}{\partial \rho_{1}} & \frac{\partial \bar{a}_{\frac{N-1}{2}}}{\partial \theta_{1}} & \cdots & \frac{\partial \bar{a}_{\frac{N-1}{2}}}{\partial \rho_{M}} & \frac{\partial \bar{a}_{\frac{N-1}{2}}}{\partial \theta_{M}} & \frac{\partial \bar{a}_{\frac{N-1}{2}}}{\partial \delta_{1}} & \cdots & \frac{\partial \bar{a}_{\frac{N-1}{2}}}{\partial \delta_{L}} \\
\frac{\partial \phi}{\partial \rho_{1}} & \frac{\partial \phi}{\partial \theta_{1}} & \cdots & \frac{\partial \phi}{\partial \rho_{M}} & \frac{\partial \phi}{\partial \theta_{M}} & \frac{\partial \phi}{\partial \delta_{1}} & \cdots & \frac{\partial \phi}{\partial \delta_{L}}
\end{array}\right| .
\end{aligned}
$$


This step was achieved in two stages: first, by adding each even row to the one above, and then by multiplying each even row by -1 and adding to it $1 / 2$ the row above.

Now note that

$$
\begin{aligned}
\frac{\partial \phi}{\partial x} & =\frac{\partial \phi}{\partial a_{N}} \frac{\partial a_{N}}{\partial x}=\frac{\partial a_{N}}{\partial x}\left(\frac{1}{i a_{N}}\right), \\
\frac{\partial a_{N-j}}{\partial x} & =a_{N} \frac{\partial \bar{a}_{j}}{\partial x}+\bar{a}_{j} \frac{\partial a_{N}}{\partial x}
\end{aligned}
$$

So we have

$$
\begin{aligned}
& J_{\mathbb{C}}=\left(-\frac{1}{2}\right)^{\frac{N-1}{2}}\left(\frac{1}{i a_{N}}\right)\left(\frac{1}{a_{N}}\right)^{\frac{N-1}{2}} \\
& \times\left|\begin{array}{cccccccc}
\frac{\partial a_{1}}{\partial \rho_{1}} & \frac{\partial a_{1}}{\partial \theta_{1}} & \cdots & \frac{\partial a_{1}}{\partial \rho_{M}} & \frac{\partial a_{1}}{\partial \theta_{M}} & \frac{\partial a_{1}}{\partial \delta_{1}} & \cdots & \frac{\partial a_{1}}{\partial \delta_{L}} \\
\frac{\partial a_{N-1}}{\partial \rho_{1}} & \frac{\partial a_{N-1}}{\partial \theta_{1}} & \cdots & \frac{\partial a_{N-1}}{\partial \rho_{M}} & \frac{\partial a_{N-1}}{\partial \theta_{M}} & \frac{\partial a_{N-1}}{\partial \delta_{1}} & \cdots & \frac{\partial a_{N-1}}{\partial \delta_{L}} \\
\vdots & \vdots & \ddots & \vdots & \vdots & \vdots & \ddots & \vdots \\
\frac{\partial a_{\frac{N-1}{2}}}{\partial \rho_{1}} & \frac{\partial a_{\frac{N-1}{2}}}{\partial \theta_{1}} & \cdots & \frac{\partial a_{\frac{N-1}{2}}}{\partial \rho_{M}} & \frac{\partial a_{\frac{N-1}{2}}}{\partial \theta_{M}} & \frac{\partial a_{\frac{N-1}{2}}}{\partial \delta_{1}} & \cdots & \frac{\partial a_{\frac{N-1}{2}}}{\partial \delta_{L}} \\
\frac{\partial a_{\frac{N+1}{2}}}{\partial \rho_{1}} & \frac{\partial a_{\frac{N+1}{2}}}{\partial \theta_{1}} & \cdots & \frac{\partial a_{\frac{N+1}{2}}}{\partial \rho_{M}} & \frac{\partial a_{\frac{N+1}{2}}}{\partial \theta_{M}} & \frac{\partial a_{\frac{N+1}{2}}}{\partial \delta_{1}} & \cdots & \frac{\partial a_{\frac{N+1}{2}}}{\partial \delta_{L}} \\
\frac{\partial a_{N}}{\partial \rho_{1}} & \frac{\partial a_{N}}{\partial \theta_{1}} & \cdots & \frac{\partial a_{N}}{\partial \rho_{M}} & \frac{\partial a_{N}}{\partial \theta_{M}} & \frac{\partial a_{N}}{\partial \delta_{1}} & \cdots & \frac{\partial a_{N}}{\partial \delta_{L}}
\end{array}\right| .
\end{aligned}
$$

So we have, where $\varepsilon$ is a quantity with modulus one that may vary at each occurrence,

$$
J_{\mathbb{C}}=\varepsilon\left(\frac{1}{2}\right)^{\frac{N-1}{2}}\left|\begin{array}{cccccccc}
\frac{\partial a_{1}}{\partial \rho_{1}} & \frac{\partial a_{1}}{\partial \theta_{1}} & \cdots & \frac{\partial a_{1}}{\partial \rho_{M}} & \frac{\partial a_{1}}{\partial \theta_{M}} & \frac{\partial a_{1}}{\partial \delta_{1}} & \cdots & \frac{\partial a_{1}}{\partial \delta_{L}} \\
\vdots & \vdots & \ddots & \vdots & \vdots & \vdots & \ddots & \vdots \\
\frac{\partial a_{N}}{\partial \rho_{1}} & \frac{\partial a_{N}}{\partial \theta_{1}} & \cdots & \frac{\partial a_{N}}{\partial \rho_{M}} & \frac{\partial a_{N}}{\partial \theta_{M}} & \frac{\partial a_{N}}{\partial \delta_{1}} & \cdots & \frac{\partial a_{N}}{\partial \delta_{L}}
\end{array}\right| .
$$

For convenience let us set $\nu_{j}=1 / \overline{\beta_{j}}$. (We thank the editor for suggesting how to improve the next part of the proof. Our argument was more lengthy.) The proof of the lemma is achieved by expressing the determinant (3.11) in terms of

$$
\begin{aligned}
& \left|\begin{array}{cccccccc}
\frac{\partial a_{1}}{\partial \beta_{1}} & \frac{\partial a_{1}}{\partial \nu_{1}} & \cdots & \frac{\partial a_{1}}{\partial \beta_{M}} & \frac{\partial a_{1}}{\partial \nu_{M}} & \frac{\partial a_{1}}{\partial \alpha_{1}} & \cdots & \frac{\partial a_{1}}{\partial \alpha_{L}} \\
\vdots & \vdots & \ddots & \vdots & \vdots & \vdots & \ddots & \vdots \\
\frac{\partial a_{N}}{\partial \beta_{1}} & \frac{\partial a_{N}}{\partial \nu_{1}} & \cdots & \frac{\partial a_{N}}{\partial \beta_{M}} & \frac{\partial a_{N}}{\partial \nu_{M}} & \frac{\partial a_{N}}{\partial \alpha_{1}} & \cdots & \frac{\partial a_{N}}{\partial \alpha_{L}}
\end{array}\right| \\
& =(-1)^{N} \Delta\left(\beta_{1}, \nu_{1}, \ldots, \beta_{M}, \nu_{M}, \alpha_{1}, \ldots, \alpha_{L}\right) .
\end{aligned}
$$

To prove this equality note that the left hand side is a homogeneous polynomial of order $N(N-1) / 2$ in the variables $\beta_{1}, \nu_{1}, \ldots, \beta_{M}, \nu_{M}, \alpha_{1}, \ldots, \alpha_{L}$. It is also antisymmetric under exchange of any two of the variables. Therefore, up to a constant, it must be proportional to the Vandermonde $\Delta\left(\beta_{1}, \nu_{1}, \ldots, \beta_{M}, \nu_{M}, \alpha_{1}, \ldots, \alpha_{L}\right)$ (defined in (1.13)). The constant $(-1)^{N}$ can be determined as follows. Let us denote by $x_{1}, \ldots, x_{N}$ the variables $\beta_{1}, \nu_{1}, \ldots$, $\beta_{M}, \nu_{M}, \alpha_{1}, \ldots, \alpha_{L}$. The generic element in the Jacobian (3.12) is

$$
\frac{\partial a_{j}}{\partial x_{k}}=(-1)^{j} e_{j-1, k}
$$

where $e_{j, k}$ is the $j$-th symmetric function in the $x_{l}$ excluding the variable $x_{k}$. We want to compute the coefficient in front of

$$
x_{1}^{N-1} x_{2}^{N-2} \cdots x_{N}
$$


Now, any $x_{j}$ in any element of the Jacobian matrix appears at most with exponent one. Furthermore, for any given choice of $j$ indices $l_{1}, l_{2}, \ldots, l_{j},\left(l_{m} \neq k\right)$ the product

$$
x_{l_{1}} x_{l_{2}} \cdots x_{l_{j}}
$$

appears once and only once in the symmetric function $e_{j, k}$. It follows that the monomial (3.14) can only come from the term

$$
\frac{\partial a_{1}}{\partial x_{1}} \frac{\partial a_{2}}{\partial x_{2}} \cdots \frac{\partial a_{N}}{\partial x_{N}}
$$

in the expansion of the Jacobian and that the coefficient in front of it is $(-1)^{N(N+1) / 2}$. The constant $(-1)^{N}$ is obtained by observing that the monomial (3.14) appear with a factor $(-1)^{N(N-1) / 2}$ in the expansion of the Vandermonde.

The relation between the determinants (3.11) and (3.12) is obtained by analytically continuing equations (3.4) in the complex planes of $\rho$ and $\theta$ and then by changing the variables from $\rho_{1}, \theta_{1}, \ldots, \rho_{M}, \theta_{M}, \delta_{1}, \ldots, \delta_{L}$ to $\beta_{1}, \nu_{1}, \ldots, \beta_{M}, \nu_{M}, \alpha_{1}, \ldots, \alpha_{L}$. Thus, we incur the further Jacobian

$$
\left|\begin{array}{cccccccc}
\frac{\partial \rho_{1}}{\partial \beta_{1}} & \frac{\partial \rho_{1}}{\partial \nu_{1}} & \cdots & \frac{\partial \rho_{1}}{\partial \beta_{M}} & \frac{\partial \rho_{1}}{\partial \nu_{M}} & \frac{\partial \rho_{1}}{\partial \alpha_{1}} & \cdots & \frac{\partial \rho_{1}}{\partial \alpha_{L}} \\
\frac{\partial \theta_{1}}{\partial \beta_{1}} & \frac{\partial \theta_{1}}{\partial \nu_{1}} & \cdots & \frac{\partial \theta_{1}}{\partial \beta_{M}} & \frac{\partial \theta_{1}}{\partial \nu_{M}} & \frac{\partial \theta_{1}}{\partial \alpha_{1}} & \cdots & \frac{\partial \theta_{1}}{\partial \alpha_{L}} \\
\vdots & \vdots & \ddots & \vdots & \vdots & \vdots & \ddots & \vdots \\
\frac{\partial \rho_{M}}{\partial \beta_{1}} & \frac{\partial \rho_{M}}{\partial \nu_{1}} & \cdots & \frac{\partial \rho_{M}}{\partial \beta_{M}} & \frac{\partial \rho_{M}}{\partial \nu_{M}} & \frac{\partial \rho_{M}}{\partial \alpha_{1}} & \cdots & \frac{\partial \rho_{M}}{\partial \alpha_{L}} \\
\frac{\partial \theta_{M}}{\partial \beta_{1}} & \frac{\partial \theta_{M}}{\partial \nu_{1}} & \cdots & \frac{\partial \theta_{M}}{\partial \beta_{M}} & \frac{\partial \theta_{M}}{\partial \nu_{M}} & \frac{\partial \theta_{M}}{\partial \alpha_{1}} & \cdots & \frac{\partial \theta_{M}}{\partial \alpha_{L}} \\
\frac{\partial \delta_{1}}{\partial \beta_{1}} & \frac{\partial \delta_{1}}{\partial \nu_{1}} & \cdots & \frac{\partial \delta_{1}}{\partial \beta_{M}} & \frac{\partial \delta_{1}}{\partial \nu_{M}} & \frac{\partial \delta_{1}}{\partial \alpha_{1}} & \cdots & \frac{\partial \delta_{1}}{\partial \alpha_{L}} \\
\vdots & \vdots & \ddots & \vdots & \vdots & \vdots & \ddots & \vdots \\
\frac{\partial \delta_{L}}{\partial \beta_{1}} & \frac{\partial \delta_{L}}{\partial \nu_{1}} & \cdots & \frac{\partial \delta_{L}}{\partial \beta_{M}} & \frac{\partial \delta_{L}}{\partial \nu_{M}} & \frac{\partial \delta_{L}}{\partial \alpha_{1}} & \cdots & \frac{\partial \delta_{L}}{\partial \alpha_{L}}
\end{array}\right| .
$$

Using equations (3.4) the computation of this determinant is straightforward. The matrix is all zero except for $2 \times 2$ blocks on the diagonal for the first $2 M$ columns, and then single elements on the diagonal for the last $L$ columns. A given $2 \times 2$ block looks like

$$
\left|\begin{array}{ll}
\frac{\partial \rho_{j}}{\partial \beta_{j}} & \frac{\partial \rho_{j}}{\partial \nu_{j}} \\
\frac{\partial \theta_{j}}{\partial \beta_{j}} & \frac{\partial \theta_{j}}{\partial \nu_{j}}
\end{array}\right|=\left|\begin{array}{cc}
\frac{1}{2 \sqrt{\beta_{j} \nu_{j}}} & -\frac{1}{2 \nu_{j}} \sqrt{\frac{\beta_{j}}{\nu_{j}}} \\
-\frac{i}{2 \beta_{j}} & -\frac{i}{2 \nu_{j}}
\end{array}\right|=-\frac{i}{2 \nu_{j} \sqrt{\beta_{j} \nu_{j}}},
$$

and the single elements on the diagonal have modulus one. So, dividing (3.12) by (3.17) we have

$$
J_{\mathbb{C}}=\varepsilon 2^{M-(N-1) / 2}\left(\prod_{m=1}^{M} \frac{1}{\rho_{m}}\right) \Delta\left(\beta_{1}, \frac{1}{\bar{\beta}_{1}}, \beta_{2}, \frac{1}{\bar{\beta}_{2}}, \ldots, \beta_{m}, \frac{1}{\bar{\beta}_{m}}, \alpha_{1}, \ldots, \alpha_{L}\right) .
$$

Here $\varepsilon$ incorporates quantities that may depend on $\beta_{1}, \frac{1}{\bar{\beta}_{1}}, \beta_{2}, \frac{1}{\bar{\beta}_{2}}, \ldots, \beta_{m}, \frac{1}{\bar{\beta}_{m}}, \alpha_{1}, \ldots, \alpha_{L}$ but has absolute value one. This completes the proof in the case $N$ is odd.

When $N$ is even, we want the Jacobian of the transformation from the real, independent variables $\operatorname{Re} a_{1}, \operatorname{Im} a_{1}, \ldots, \operatorname{Re} a_{\frac{N}{2}}, \operatorname{Im} a_{\frac{N}{2}}$ to the real independent variables $\rho_{1}, \theta_{1}, \rho_{2}, \theta_{2}, \ldots$, $\rho_{M}, \theta_{M}, \delta_{1}, \ldots, \delta_{L}$. 
So, we start with

$$
\begin{aligned}
& J_{\mathbb{C}}=\left|\begin{array}{cccccccc}
\frac{\partial \operatorname{Re} a_{1}}{\partial \rho_{1}} & \frac{\partial \operatorname{Re} a_{1}}{\partial \theta_{1}} & \ldots & \frac{\partial \operatorname{Re} a_{1}}{\partial \rho_{M}} & \frac{\partial \operatorname{Re} a_{1}}{\partial \theta_{M}} & \frac{\partial \operatorname{Re} a_{1}}{\partial \delta_{1}} & \ldots & \frac{\partial \operatorname{Re} a_{1}}{\partial \delta_{L}} \\
\frac{\partial \operatorname{Im} a_{1}}{\partial \rho_{1}} & \frac{\partial \operatorname{Im} a_{1}}{\partial \theta_{1}} & \ldots & \frac{\partial \operatorname{Im} a_{1}}{\partial \rho_{M}} & \frac{\partial \operatorname{Im} a_{1}}{\partial \theta_{M}} & \frac{\partial \operatorname{Im} a_{1}}{\partial \delta_{1}} & \ldots & \frac{\partial \operatorname{Im} a_{1}}{\partial \delta_{L}} \\
\vdots & \vdots & \ddots & \vdots & \vdots & \vdots & \ddots & \vdots \\
\frac{\partial \operatorname{Re} a_{\frac{N}{2}}}{\partial \rho_{1}} & \frac{\partial \operatorname{Re} a_{\frac{N}{2}}}{\partial \theta_{1}} & \ldots & \frac{\partial \operatorname{Re} a_{\frac{N}{2}}}{\partial \rho_{M}} & \frac{\partial \operatorname{Re} a_{\frac{N}{2}}}{\partial \theta_{M}} & \frac{\partial \operatorname{Re} a_{\frac{N}{2}}}{\partial \delta_{1}} & \ldots & \frac{\partial \operatorname{Re} a_{\frac{N}{2}}}{\partial \delta_{L}} \\
\frac{\partial \operatorname{Im} a_{\frac{N}{2}}}{\partial \rho_{1}} & \frac{\partial \operatorname{Im} a_{\frac{N}{2}}}{\partial \theta_{1}} & \ldots & \frac{\partial \operatorname{Im} a_{\frac{N}{2}}}{\partial \rho_{M}} & \frac{\partial \operatorname{Im} a_{\frac{N}{2}}}{\partial \theta_{M}} & \frac{\partial \operatorname{Im} a_{\frac{N}{2}}}{\partial \delta_{1}} & \ldots & \frac{\partial \operatorname{Im} a_{\frac{N}{2}}}{\partial \delta_{L}}
\end{array}\right| \\
& =\left(-\frac{1}{2}\right)^{\frac{N}{2}}\left|\begin{array}{cccccccc}
\frac{\partial a_{1}}{\partial \rho_{1}} & \frac{\partial a_{1}}{\partial \theta_{1}} & \cdots & \frac{\partial a_{1}}{\partial \rho_{M}} & \frac{\partial a_{1}}{\partial \theta_{M}} & \frac{\partial a_{1}}{\partial \delta_{1}} & \cdots & \frac{\partial a_{1}}{\partial \delta_{L}} \\
\frac{\partial \bar{a}_{1}}{\partial \rho_{1}} & \frac{\partial \bar{a}_{1}}{\partial \theta_{1}} & \cdots & \frac{\partial \bar{a}_{1}}{\partial \rho_{M}} & \frac{\partial \bar{a}_{1}}{\partial \theta_{M}} & \frac{\partial \bar{a}_{1}}{\partial \delta_{1}} & \cdots & \frac{\partial \bar{a}_{1}}{\partial \delta_{L}} \\
\vdots & \vdots & \ddots & \vdots & \vdots & \vdots & \ddots & \vdots \\
\frac{\partial a_{\frac{N}{2}}}{\partial \rho_{1}} & \frac{\partial a_{\frac{N}{2}}}{\partial \theta_{1}} & \cdots & \frac{\partial a_{\frac{N}{2}}}{\partial \rho_{M}} & \frac{\partial a_{\frac{N}{2}}}{\partial \theta_{M}} & \frac{\partial a_{\frac{N}{2}}}{\partial \delta_{1}} & \cdots & \frac{\partial a_{\frac{N}{2}}}{\partial \delta_{L}} \\
\frac{\partial \bar{a}_{\frac{N}{2}}}{\partial \rho_{1}} & \frac{\partial \bar{a}_{\frac{N}{2}}}{\partial \theta_{1}} & \cdots & \frac{\partial \bar{a}_{\frac{N}{2}}}{\partial \rho_{M}} & \frac{\partial \bar{a}_{\frac{N}{2}}}{\partial \theta_{M}} & \frac{\partial \bar{a}_{\frac{N}{2}}}{\partial \delta_{1}} & \cdots & \frac{\partial \bar{a}_{\frac{N}{2}}}{\partial \delta_{L}}
\end{array}\right| .
\end{aligned}
$$

Now we want to transform the derivatives of $\bar{a}_{j}$ into derivatives of $a_{N-j}$, with the exception of $\bar{a}_{N / 2}$, which should give us derivatives of $a_{N}$. Note that $a_{N}$ has modulus 1 and

$$
\frac{a_{N / 2}}{\bar{a}_{N / 2}}=a_{N}
$$

Therefore, we have

$$
\begin{aligned}
\frac{\partial a_{N}}{\partial x} & =\frac{\partial}{\partial x}\left(\frac{a_{N / 2}}{\bar{a}_{N / 2}}\right) \\
& =\frac{1}{\bar{a}_{N / 2}} \frac{\partial a_{N / 2}}{\partial x}-\frac{a_{N / 2}}{\left(\bar{a}_{N / 2}\right)^{2}} \frac{\partial \bar{a}_{N / 2}}{\partial x} .
\end{aligned}
$$

Thus we need to multiply the last row of (3.20) by $-a_{N / 2} /\left(\bar{a}_{N / 2}\right)^{2}$ and add to it $1 / \bar{a}_{N / 2}$ times the row above. This procedure has multiplied the determinant by a factor $-a_{N / 2} /\left(\bar{a}_{N / 2}\right)^{2}=$ $-a_{N} / \bar{a}_{N / 2}$. Note that $a_{N}$ has modulus one, but $a_{N / 2}$ does not. Thus, incorporating factors of modulus one into $\varepsilon$, the Jacobian is

$$
J_{\mathbb{C}}=\varepsilon \bar{a}_{N / 2}\left(\frac{1}{2}\right)^{\frac{N}{2}}\left|\begin{array}{cccccccc}
\frac{\partial a_{1}}{\partial \rho_{1}} & \frac{\partial a_{1}}{\partial \theta_{1}} & \cdots & \frac{\partial a_{1}}{\partial \rho_{M}} & \frac{\partial a_{1}}{\partial \theta_{M}} & \frac{\partial a_{1}}{\partial \delta_{1}} & \cdots & \frac{\partial a_{1}}{\partial \delta_{L}} \\
\vdots & \vdots & \ddots & \vdots & \vdots & \vdots & \ddots & \vdots \\
\frac{\partial a_{N}}{\partial \rho_{1}} & \frac{\partial a_{N}}{\partial \theta_{1}} & \cdots & \frac{\partial a_{N}}{\partial \rho_{M}} & \frac{\partial a_{N}}{\partial \theta_{M}} & \frac{\partial a_{N}}{\partial \delta_{1}} & \cdots & \frac{\partial a_{N}}{\partial \delta_{L}}
\end{array}\right| .
$$

It is now just necessary to follow the steps as in the odd $N$ case in order to prove Lemma 3.1 .

\section{Calculation of the Jacobian: Real Case}

We prove Lemma 2.2. It would be possible to cover a more general case, considering the number of zeros on the unit circle, the number in complex conjugate pairs located symmetrically with respect to the unit circle, and the number of real zeros. But our concern here is with the case that all zeros are on the unit circle so we only consider the case we require in this paper. We describe the odd degree case in detail and then discuss the modifications required for the even degree case. 
We have a self-reciprocal polynomial $f(z)$ that has a root at -1 and at $\beta_{1}, \bar{\beta}_{1}, \ldots, \beta_{M}, \bar{\beta}_{M}$ on the unit circle. Thus, $\bar{\beta}_{j}=1 / \beta_{j}$. The polynomial $f(z)$ has order $N=2 M+1$ :

$$
\begin{aligned}
f(z) & =(z+1)\left(z-\beta_{1}\right)\left(z-\bar{\beta}_{1}\right) \cdots\left(z-\beta_{M}\right)\left(z-\bar{\beta}_{M}\right) \\
& =z^{N}+a_{1} z^{N-1}+a_{2} z^{N-2}+\cdots+a_{N-2} z^{2}+a_{N-1} z+1 .
\end{aligned}
$$

We have the functional equation

$$
f(z)=z^{N} f\left(\frac{1}{z}\right)
$$

which implies the symmetry of the coefficients

$$
a_{n}=a_{N-n} .
$$

So the polynomial is determined by $a_{1}, \ldots, a_{M}$. We also have

$$
a_{n}=(-1)^{n} e_{n}\left(-1, \beta_{1}, \bar{\beta}_{1}, \ldots, \beta_{M}, \bar{\beta}_{M}\right),
$$

where $e_{n}$ is the $n$th elementary symmetric function.

We want the Jacobian of the transformation from the real variables $a_{1}, \ldots, a_{M}$ to the real variables $t_{1}, \ldots, t_{M}$, where $\beta_{j}=e^{i t_{j}}$.

We start with

$$
J_{\mathbb{R}}:=\left|\begin{array}{cccc}
\frac{\partial a_{1}}{\partial t_{1}} & \frac{\partial a_{1}}{\partial t_{2}} & \cdots & \frac{\partial a_{1}}{\partial t_{M}} \\
\vdots & \vdots & \ddots & \vdots \\
\frac{\partial a_{M}}{\partial t_{1}} & \frac{\partial a_{M}}{\partial t_{2}} & \cdots & \frac{\partial a_{M}}{\partial t_{M}}
\end{array}\right|=i^{M} \beta_{1} \cdots \beta_{M}\left|\begin{array}{cccc}
\frac{\partial a_{1}}{\partial \beta_{1}} & \frac{\partial a_{1}}{\partial \beta_{2}} & \cdots & \frac{\partial a_{1}}{\partial \beta_{M}} \\
\vdots & \vdots & \ddots & \vdots \\
\frac{\partial a_{M}}{\partial \beta_{1}} & \frac{\partial a_{M}}{\partial \beta_{2}} & \cdots & \frac{\partial a_{M}}{\partial \beta_{M}}
\end{array}\right|,
$$

where we used the fact that

$$
\frac{\partial a_{j}}{\partial t_{k}}=\frac{\partial a_{j}}{\partial \beta_{k}} \frac{d \beta_{k}}{d t_{k}}=i \beta_{k} \frac{\partial a_{j}}{\partial \beta_{k}} .
$$

At this point our calculation becomes somewhat different than the complex case in the previous section. It is possible that similar methods could be used here, but the argument would be more sophisticated because (as we will see), the answer is not symmetric in all the variables. We will directly calculate the Jacobian, instead of deducing the form of the answer from its various symmetries.

Following the method in [26], for each $m$ we have

$$
a_{n}=(-1)^{n}\left(\left(\beta_{m}+\frac{1}{\beta_{m}}\right) e_{n-1, m}^{\prime}+e_{n-2, m}^{\prime}+e_{n, m}^{\prime}\right),
$$

where we write $e_{n, m}^{\prime}$ for the $n$th symmetric function in -1 and all the $\beta_{j}$ and $\bar{\beta}_{j}=\frac{1}{\beta_{j}}, j=$ $1, \ldots, M$, except for $\beta_{m}$ and $\frac{1}{\beta_{m}}$, with the convention that $e_{0}=e_{0, m}^{\prime}=1$ and $e_{n}=e_{n, m}^{\prime}=0$ if $n$ is greater than the number of variables. Thus, we obtain

$$
\frac{\partial a_{n}}{\partial \beta_{m}}=(-1)^{n}\left(\left(1-\frac{1}{\beta_{m}^{2}}\right) e_{n-1, m}^{\prime}\right)
$$

so our determinant is

$$
J_{\mathbb{R}}=\varepsilon \prod_{m=1}^{M}\left(\beta_{m}-\frac{1}{\beta_{m}}\right)\left|\begin{array}{cccc}
e_{0,1}^{\prime} & e_{0,2}^{\prime} & \cdots & e_{0, M}^{\prime} \\
e_{1,1}^{\prime} & e_{1,2}^{\prime} & \cdots & e_{1, M}^{\prime} \\
\vdots & \vdots & \ddots & \vdots \\
e_{M-1,1}^{\prime} & e_{M-1,2}^{\prime} & \cdots & e_{M-1, M}^{\prime}
\end{array}\right|
$$


Here and following we use $\varepsilon$ to denote a number with absolute value 1 , which may be different at each occurrence.

Now we define the following polynomials:

$$
\begin{aligned}
f_{\ell}(x) & =(x+1) \prod_{\substack{m=1 \\
m \neq \ell}}^{M}\left(x-\beta_{m}\right)\left(x-\frac{1}{\beta_{m}}\right)=\sum_{n=0}^{N-2}(-1)^{n} e_{n, \ell}^{\prime} x^{N-2-n} \\
& =\sum_{n=0}^{M-1}(-1)^{n} e_{n, \ell}^{\prime}\left(x^{N-2-n}+x^{n}\right) \\
& =x^{M-\frac{1}{2}} \sum_{n=0}^{M-1}(-1)^{n} e_{n, \ell}^{\prime}\left(x^{M-\frac{1}{2}-n}+x^{-M+\frac{1}{2}+n}\right),
\end{aligned}
$$

where the next-to-last step used the fact that $e_{n, \ell}^{\prime}=-e_{N-2-n, \ell}^{\prime}$, since the roots come in conjugate pairs except for the extra root at -1 .

Note that $f_{\ell}\left(\beta_{m}\right)=0$ if $\ell \neq m$. Also we have

$$
f_{\ell}\left(e^{i t}\right)=2 e^{i\left(M-\frac{1}{2}\right) t} \sum_{n=0}^{M-1}(-1)^{n} e_{n, \ell}^{\prime} \cos \left(\left(M-\frac{1}{2}-n\right) t\right) .
$$

Therefore, with

$$
\begin{gathered}
V\left(t_{1}, \ldots, t_{M}\right)= \\
\left|\begin{array}{ccccc}
\cos \left(\left(M-\frac{1}{2}\right) t_{1}\right) & -\cos \left(\left(M-\frac{3}{2}\right) t_{1}\right) & \cdots & (-1)^{M-2} \cos \left(\frac{3}{2} t_{1}\right) & (-1)^{M-1} \cos \left(\frac{1}{2} t_{1}\right) \\
\cos \left(\left(M-\frac{1}{2}\right) t_{2}\right) & -\cos \left(\left(M-\frac{3}{2}\right) t_{2}\right) & \cdots & (-1)^{M-2} \cos \left(\frac{3}{2} t_{2}\right) & (-1)^{M-1} \cos \left(\frac{1}{2} t_{2}\right) \\
\vdots & \vdots & \ddots & \vdots & \vdots \\
\cos \left(\left(M-\frac{1}{2}\right) t_{M}\right) & -\cos \left(\left(M-\frac{3}{2}\right) t_{M}\right) & \cdots & (-1)^{M-2} \cos \left(\frac{3}{2} t_{M}\right) & (-1)^{M-1} \cos \left(\frac{1}{2} t_{M}\right)
\end{array}\right|
\end{gathered}
$$

we have

$$
V\left(t_{1}, \ldots, t_{M}\right) J_{\mathbb{R}}=C_{M}\left|\begin{array}{cccc}
f_{1}\left(\beta_{1}\right) & 0 & \cdots & 0 \\
0 & f_{2}\left(\beta_{2}\right) & \cdots & 0 \\
\vdots & \vdots & \ddots & \vdots \\
0 & 0 & \cdots & f_{M}\left(\beta_{M}\right)
\end{array}\right|
$$

where

$$
C_{M}=\varepsilon 2^{-M} \prod_{m=1}^{M}\left(\beta_{m}-\frac{1}{\beta_{m}}\right)
$$

Since

$$
2 \sin \left(\frac{1}{2} t\right) \cos \left(\left(M-\frac{1}{2}-n\right) t\right)=\sin ((M-n) t-\sin ((M-1-n) t),
$$

by multiplying each row by $\sin \left(\frac{1}{2} t_{n}\right)$ we have that

$$
V\left(t_{1}, \ldots t_{M}\right) \cdot 2^{M} \prod_{n} \sin \left(\frac{1}{2} t_{n}\right)
$$


equals a determinant whose rows are

$$
\left(\sin \left(M t_{j}\right)-\sin \left((M-1) t_{j}\right) \quad \cdots \quad \sin \left(2 t_{j}\right)-\sin \left(t_{j}\right) \quad \sin \left(t_{j}\right)\right) .
$$

By elementary column operations starting with the last column, this equals a determinant with rows

$$
\left(\sin \left(M t_{j}\right) \quad \sin \left((M-1) t_{j}\right) \quad \cdots \quad \sin \left(2 t_{j}\right) \quad \sin \left(t_{j}\right)\right)
$$

Since

$$
\sin ((n+1) t)=\sin (t)\left(2^{n} \cos ^{n}(t)+\text { lower order terms in } \cos (t)\right),
$$

by elementary column operations we find that the determinant whose rows are (4.18) equals a determinant with rows

$$
\sin \left(t_{j}\right) \times\left(\begin{array}{lllll}
2^{M-1} \cos ^{M-1}\left(t_{j}\right) & 2^{M-2} \cos ^{M-2}\left(t_{j}\right) & \ldots & 2 \cos \left(t_{j}\right) & 1
\end{array}\right) .
$$

Since this last determinant is a Vandermonde, we have shown

$$
\begin{aligned}
V\left(t_{1}, \ldots t_{M}\right) & =2^{M(M-3) / 2} \prod_{n} \frac{\sin \left(t_{n}\right)}{\sin \left(\frac{1}{2} t_{n}\right)} \Delta\left(\cos \left(t_{1}\right), \ldots, \cos \left(t_{M}\right)\right) \\
& =2^{M(M-1) / 2} \prod_{n} \cos \left(\frac{1}{2} t_{n}\right) \Delta\left(\cos \left(t_{1}\right), \ldots, \cos \left(t_{M}\right)\right) .
\end{aligned}
$$

Combining (4.13), (4.14), and (4.21) we have

$$
J_{\mathbb{R}}=\varepsilon 2^{-M(M+1) / 2} \frac{\prod_{m}\left(\beta_{m}-\bar{\beta}_{m}\right) \prod_{m} f_{m}\left(\beta_{m}\right)}{\prod_{m} \cos \left(\frac{1}{2} t_{m}\right) \Delta\left(\cos \left(t_{1}\right), \ldots, \cos \left(t_{M}\right)\right)} .
$$

Since,

$$
\begin{aligned}
\prod_{m} f_{m}\left(\beta_{m}\right) & =\prod_{m}\left(\beta_{m}+1\right) \prod_{k \neq m}\left(\beta_{m}-\beta_{k}\right)\left(\beta_{m}-\bar{\beta}_{k}\right) \\
& =2^{2 M^{2}-M} \prod_{m} \cos \left(\frac{1}{2} t_{m}\right) \prod_{k \neq m} \sin \left(\frac{t_{m}-t_{k}}{2}\right) \sin \left(\frac{t_{m}+t_{k}}{2}\right),
\end{aligned}
$$

and

$$
\begin{aligned}
\Delta\left(\cos t_{1}, \ldots, \cos t_{M}\right) & =\prod_{j<k}\left(\cos t_{k}-\cos t_{j}\right) \\
& =2^{M(M-1) / 2} \prod_{j<k} \sin \left(\frac{t_{j}-t_{k}}{2}\right) \sin \left(\frac{t_{j}+t_{k}}{2}\right),
\end{aligned}
$$

and $\beta_{m}-\bar{\beta}_{m}=2 i \sin \left(t_{m}\right)$, we find that,

$$
\begin{aligned}
J_{\mathbb{R}} & =\varepsilon 2^{M^{2}} \prod_{m} \sin \left(t_{m}\right) \prod_{j<k} \sin \left(\frac{t_{k}-t_{j}}{2}\right) \sin \left(\frac{t_{k}+t_{j}}{2}\right) \\
& =\varepsilon \prod_{m}\left(\beta_{m}-\bar{\beta}_{m}\right) \prod_{j<k}\left(\beta_{k}-\beta_{j}\right)\left(\beta_{k}-\bar{\beta}_{j}\right),
\end{aligned}
$$

as claimed. 
In the case of even degree, only a few modifications are needed. The polynomials (4.10) are replaced by

$$
\begin{aligned}
f_{\ell}(x) & =\prod_{\substack{m=1 \\
m \neq \ell}}^{M}\left(x-\beta_{m}\right)\left(x-\frac{1}{\beta_{m}}\right) \\
& =x^{M-1}\left(\sum_{n=0}^{M-2}(-1)^{n} e_{n, \ell}^{\prime}\left(x^{M-1-n}+x^{-M+1+n}\right)+(-1)^{M-1} e_{M-1, \ell}^{\prime}\right),
\end{aligned}
$$

so

$$
f_{\ell}\left(e^{i t}\right)=\varepsilon\left(2 \sum_{n=0}^{M-2}(-1)^{n} e_{n, \ell}^{\prime} \cos ((M-1-n) t)+(-1)^{M-1} e_{M-1, \ell}^{\prime}\right) .
$$

The determinant $V\left(t_{1}, \ldots, t_{M}\right)$ has entries $\cos \left((M-1-n) t_{j}\right)$, except for the last column, where $n=M-1$, which is multiplied by a factor of $\frac{1}{2}$. Since

$$
\cos (n t)=2^{n-1} \cos ^{n}(t)+\text { lower order terms in } \cos (t),
$$

we recognize $V\left(t_{1}, \ldots, t_{M}\right)$ as a Vandermonde, which is $2^{M}$ times smaller than the Vandermonde which appeared in the odd degree case. The only other differences in the calculation is to omit the factor $2^{M} \prod \sin \left(t_{n} / 2\right)$ from (4.16) and $\sin \left(t_{j}\right)$ from (4.20). The overall effect of those factors is to multiply the Jacobian by

$$
\prod_{n} \frac{\sin \left(t_{n}\right)}{2 \sin \left(\frac{t_{n}}{2}\right)}=\prod_{n} \cos \left(\frac{t_{n}}{2}\right) .
$$

That factor replaces $\prod_{m}\left(\beta_{m}+1\right)=2^{M} \prod_{m} \cos \left(\frac{1}{2} t_{m}\right)$ which is now omitted from $\prod_{m} f_{m}\left(\beta_{m}\right)$, the power of 2 making up for the factor of $2^{M}$ missing from $V\left(t_{1}, \ldots, t_{M}\right)$. So the end result is the exact same formula for the Jacobian.

\section{Appendix A. Epstein zeta FunCtions ASSOCIATED to BINARY QUADRATIC FORMS.}

An example ensemble of Dirichlet series with functional equation is the Epstein zetafunctions associated to a binary quadratic form. These functions have functional equations but no Euler product. Indeed it is conjectured (Sarnak's rigidity conjecture) that there does not exist a nontrivial continuous family of Dirichlet series with functional equation and Euler product.

To create an Epstein zeta-function, begin with a binary quadratic form

$$
Q(m, n)=a m^{2}+b m n+c n^{2} .
$$

We assume that $Q$ is positive-definite, meaning that $Q(m, n)>0$ if $(m, n) \neq(0,0)$. This is equivalent to $a>0$ and $\Delta=b^{2}-4 a c<0$. In number theory one considers the case of $a, b, c \in \mathbb{Z}$, but we will allow $a, b, c$ to be arbitrary real numbers.

The Epstein zeta-function associated to $Q$ is defined as

$$
L_{Q}(s):=\sum_{m, n}^{\prime} \frac{1}{Q(m, n)^{s}},
$$

where the notation $\sum^{\prime}$ means that we sum over all pairs of intgers $(m, n)$ except for $(0,0)$. This series converges for $\operatorname{Re}(s)>1$, and $L_{Q}(s)$ has a meromorphic continuation to the entire 
complex plane except for a simple pole at $s=1$. The Epstein zeta-function satisfies the functional equation

$$
\left(\frac{\sqrt{|\Delta|}}{2 \pi}\right)^{s} \Gamma(s) L_{Q}(s)=\left(\frac{\sqrt{|\Delta|}}{2 \pi}\right)^{1-s} \Gamma(1-s) L_{Q}(1-s),
$$

where $\Gamma(s)$ is the Gamma function. Thus, $L_{Q}(s)$ has a functional equation similar to that of the Riemann $\zeta$-function and other $L$-functions of number theory. For certain quadratic forms the Epstein zeta-function can be expressed in terms of $L$-functions. For example,

$$
L_{m^{2}+n^{2}}(s)=4 \zeta(s) L\left(s, \chi_{4}\right),
$$

where $\zeta(s)$ is the Riemann zeta-function and $L\left(s, \chi_{4}\right)$ is the Dirichlet $L$-function for the nontrivial character modulo 4. This Epstein zeta function is expected to satisfy the Riemann hypothesis, but note that its zeros are a superposition of two independent sets of zeros and so will not display the characteristic quadratic repulsion expected for $L$-functions with functional equation and Euler product. For the Epstein zeta function (A4), and a handful of other special cases, $L_{Q}(s)$ has an Euler product. In the case that $a, b, c$ are integers, $L_{Q}(s)$ is a finite linear combination of Dirichlet $L$-functions. However, for generic $Q(m, n)$ there is no arithmetic structure to the Epstein zeta-function $L_{Q}(s)$, and hence no Euler product, and it is these "random Dirichlet series with functional equation" which illustrate that we are not studying an empty set of functions.

There is a natural way to parametrize the Epstein zeta-functions. Write

$$
L_{Q}(s)=a^{-s} \sum_{m, n}^{\prime} \frac{1}{|m+z n|^{2 s}},
$$

where $z=x+i y$ is a complex number satisfying $|z|^{2}=c / a$ and $2 x=b / a$. Note that the conditions on $a, b, c$ guarantee that such a number $z$ exists, and $z$ is uniquely determined if we also assume $y>0$. The above formula for $L_{Q}$ allows us to relate the Epstein zeta-function to an Eisenstein series on $S L(2, \mathbb{Z})$ :

$$
E(z ; s):=\frac{1}{2} \sum_{m, n}^{\prime} \frac{y^{s}}{|m z+n|^{2 s}} .
$$

Specifically, we have $L_{Q}(s)=2(a y)^{-s} E(z ; s)$.

The Eisenstein series $E(z ; s)$ is a well-understood object from number theory. See 12 for details. However, in that theory one usually fixes $s$ and considers $E(z ; s)$ as a function of $z$. Indeed, as a function of $z$ it has many fascinating properties: it is an eigenfunction of the hyperbolic Laplacian, and it is invariant (up to an automorphic factor) under the linearfractional action of $S L(2, \mathbb{Z})$ on the upper half-plane. Thus, the Epstein zeta-functions associated to a binary quadratic form are parametrized by the points in a fundamental domain for $S L(2, \mathbb{Z})$ :

$$
\mathcal{F}=\left\{z=x+i y:-\frac{1}{2}<x \leq \frac{1}{2}, y>0,|z|>1\right\} .
$$

In [9] evidence is given that such functions can be modeled by random trigonometric polynomials. 


\section{REFERENCES}

[1] M.V. Berry and J.P. Keating, The Riemann zeros and eigenvalue asymptotics, SIAM Rev., 41(2):236266, 1999.

[2] E. Bogomolny, O. Bohigas and P. Leboeuf, Quantum chaotic dynamics and random polynomials, J. Stat. Phys., 85:639-679, 1996

[3] J.B. Conrey and D.W. Farmer, Mean values of $L$-functions and symmetry, Int. Math. Res. Notices, 17:883-908, 2000, arXiv math.nt/9912107.

[4] J.B. Conrey, D.W. Farmer, J.P. Keating, M.O. Rubinstein, and N.C. Snaith, Integral moments of $L$ functions, Proc. Lond. Math. Soc., 91(1):33-104, 2005, arXiv math.nt/0206018

[5] J.E.A. Dunnage, The number of real zeros of a random trigonometric polynomial, Proc. Lond. Math. Soc. 16(3):53-84, 1966

[6] K. Farahmand, Topics in Random Polynomials, Addison Wesley, London 1998

[7] D.W. Farmer and S. Lemurell, Deformations of Maass forms, Math. Comp. 74(252): 1967-1982, 2005

[8] D.W. Farmer and S. Lemurell, Maass forms and their L-functions, preprint, 2005, arXiv math.NT/0506102

[9] D.W. Farmer and S. Koutsoliotas, Epstein zeta functions and random polynomials, in preparation.

[10] D.A. Hejhal, Zeros of Epstein zeta functions and supercomputers. Proceedings of the International Congress of Mathematicians, Vol. 1, 2 (Berkeley, Calif., 1986), 1362-1384, Amer. Math. Soc., Providence, RI, 1987.

[11] D.A. Hejhal, On the triple correlation of zeros of the zeta function, Inter. Math. Res. Notices, 7:293-302, 1994.

[12] H. Iwaniec, Spectral Methods of Automorphic Forms, GSM 53, American Mathematical Society, 2002.

[13] C.P. Hughes, J.P. Keating, and N. O'Connell, Random matrix theory and the derivative of the Riemann zeta function, Proc. R. Soc. Lond. A, 456:2611-2627, 2000.

[14] H. Iwaniec, W. Luo, and P. Sarnak, Low lying zeros of families of L-functions, Inst. Hautes tudes Sci. Publ. Math. No. 91 (2000), 55-131.

[15] N.M. Katz and P. Sarnak, Random Matrices, Frobenius Eigenvalues and Monodromy, American Mathematical Society Colloquium Publications, 45. American Mathematical Society, Providence, Rhode Island, 1999.

[16] N.M. Katz and P. Sarnak, Zeros of zeta functions and symmetry, Bull. Amer. Math. Soc., 36:1-26, 1999.

[17] J.P. Keating, The Riemann zeta function and quantum chaology, In Quantum Chaos; editors, G. Casati, I Guarneri, and U. Smilansky, pages 145-85. North-Holland, Amsterdam, 1993.

[18] J.P. Keating and N.C. Snaith, Random matrix theory and $L$-functions at $s=1 / 2$, Commun. Math. Phys, 214:91-110, 2000.

[19] J.P. Keating and N.C. Snaith, Random matrix theory and $\zeta(1 / 2+i t)$, Commun. Math. Phys., 214:57-89, 2000.

[20] M.L. Mehta, Random Matrices, Academic Press, London, second edition, 1991.

[21] H.L. Montgomery, The pair correlation of the zeta function, Proc. Symp. Pure Math, 24:181-93, 1973.

[22] A.M. Odlyzko, The $10^{20}$ th zero of the Riemann zeta function and 70 million of its neighbors, Preprint, 1989, http://www.dtc.umn.edu/ odlyzko/unpublished/index.html

[23] K. Petersen and C. Sinclair Conjugate reciprocal polynomials with all roots on the unit circle, preprint, 2005, arXiv math.NT/0511397.

[24] M. Rubinstein, Evidence for a Spectral Interpretation of Zeros of L-functions, PhD thesis, Princeton University, 1998.

[25] Z. Rudnick and P. Sarnak, Zeros of principal $L$-functions and random matrix theory, Duke Mathematical Journal, 81(2):269-322, 1996.

[26] C. Sinclair, Multiplicative Distance Functions, PhD thesis, The University of Texas at Austin, 2005.

[27] H. J. Sommers, F. Haake and J. Weber, Joint densities of secular coefficients for unitary matrices, $J$. Phys. A: Math. Gen., 31: 4395-4401, 1998

[28] H. Weyl, Classical Groups, Princeton University Press, 1946. 
American Institute of Mathematics

360 Portage Ave.

Palo Alto, CA 94306

USA

FARMER@AIMATH.ORG

School of Mathematics

UNIVERSITY OF BRISTOL

BRISTOL BS8 1TW

UNITED KINGDOM

F.MEZZADRI@BRISTOL.AC.UK

N.C.SNAITH@BRISTOL.AC.UK 